\title{
A comparison of transoral vestibular and bilateral areolar endoscopic thyroidectomy approaches for unilateral papillary thyroid microcarcinomas
}

\author{
Zhiliang $\mathrm{Xu}^{1}$, Junlong Song ${ }^{1}$, Yuan Wang ${ }^{1}$, Lingzhen $\operatorname{Tan}^{1}$, Shengrong Sun ${ }^{1}$, Yanyan Meng ${ }^{2}$ \\ ${ }^{1}$ Department of Breast and Thyroid Surgery, Renmin Hospital of Wuhan University, Wuhan, China \\ ${ }^{2}$ Department of Cardiology, Renmin Hospital of Wuhan University, Wuhan, China
}

Videosurgery Miniinv 2019; 14 (4): 501-508

DOI: https://doi.org/10.5114/wiitm.2019.84759

\begin{abstract}
Introduction: A transoral (TO) vestibular approach is a new remote access approach to avoid cutaneous scars in an endoscopic thyroidectomy (ET). And transoral endoscopic thyroidectomy (TOET) has been widely used in papillary thyroid microcarcinomas (PTMCS).

Aim: To evaluate and compare endoscopic thyroidectomies with central neck dissection using a bilateral areolar (BA) approach and TO approach in patients with unilateral PTMCs.

Material and methods: In total, 92 papillary thyroid cancer (PTC) patients undergoing an endoscopic unilateral thyroidectomy with central neck dissection via a TO $(n=48)$ or BA $(n=44)$ approach were retrospectively enrolled from August 2017 to December 2018. Differences in various factors, such as clinical characteristics, surgery-related parameters, including operative complications, and hospital durations and costs, were compared.

Results: No significant between-group differences in the age and sex of the patients or the side, body mass index, total operative time, operative bleeding, superior parathyroid preservation in situ, operative complications, and postoperative day of discharge were found (all $p>0.05$ ). The mean central neck dissection time, amount of postoperative drainage, extubation time, and inferior parathyroid preservation in situ were lower in the TO group as compared with these parameters in the BA group (all $p<0.05$ ). The number of central lymph nodes resected and hospitalization costs were higher in the TO group as compared with those in the BA group (all $p<0.05$ ).

Conclusions: An ET via the TO approach may be a good choice for unilateral PTMCs because of the shorter central neck dissection time, reduced postoperative drainage, better cosmetic satisfaction, and higher number of lymph nodes removed.
\end{abstract}

Key words: transoral, bilateral areola, endoscopic thyroidectomy, papillary thyroid cancer, central neck dissection.

\section{Introduction}

Thyroid cancer is the most common endocrine malignancy, with papillary thyroid cancer (PTC) being the most prevalent type $[1,2]$. With advances in ultrasonography and fine needle aspiration biopsy, the detection rate of papillary thyroid microcarcinomas (PTMCs) (i.e., a tumor of $<1 \mathrm{~cm}$ ) has markedly increased $[3,4]$. Controversy surrounds the opti- mal treatment for PTMCs. According to the extent of resection, potential surgical approaches can be divided into total thyroidectomy (TT), hemithyroidectomy, and thyroid lobectomy (TL). Although TT was thought to be the most effective for PTMCs [5], a recent study concluded that TT was not mandatory for low-risk patients and that morbidity rates associated with hypoparathyroidism and transient nerve

Address for correspondence

Yanyan Meng, Department of Cardiology, Renmin Hospital of Wuhan University, Wuhan, China, e-mail: 104366583@qq.com 
palsy were significantly higher in TT than TL patients [6]. In China, a TL is widely used in clinical practice for unilateral PTMCs.

Conventional thyroidectomy is the standard procedure for thyroid carcinoma patients. This type of surgery inevitably leaves an incisional scar in the lower anterior neck. The scar may be a cause of aesthetic concern among PTC patients, most of whom are young or middle-aged women who are concerned about the cosmetic appearance of the scar [7]. With the development of endoscopic thyroid surgical techniques, "scarless" extra-neck approaches (e.g., anterior chest, bilateral areolar (BA), and axilla) have been widely used in clinical practice [8-10]. In recent years, a transoral endoscopic thyroidectomy (TOET) approach has been widely promoted due to the absence of a scar on the entire body surface.

\section{Aim}

The aim of this study was to retrospectively evaluate and compare the TOET approach in terms of complete resection of the thyroid cancer lobe, central lymph nodes, and isthmus with the conventional BA approach in patients with unilateral PTMCs and to determine the advantages, disadvantages, and selection criteria of each approach.

\section{Material and methods}

A retrospective review was conducted of the records of 92 consecutive patients with PTMCs who underwent an endoscopic unilateral thyroidectomy with central neck dissection from August 2017 to December 2018. The patients were diagnosed with PTC by preoperative fine needle aspiration cytology. The patients were divided into two groups, depending on the endoscopic thyroidectomy (ET) approach: a TO group $(n=48)$ and a BA group $(n=44)$. Patients who met one or more of the following criteria were excluded: (1) a history of neck surgery or radiotherapy, (2) evidence of local invasion or involvement of the recurrent laryngeal nerve (RLN), and (3) preoperative imaging revealing enlarged and fused lymph nodes.

It was assumed that all the patients desired "scarless" neck surgery for aesthetic reasons. All the patients were informed about the two approaches in detail and then chose the approach according to their own wishes. The technique used was standardized, as reported in the literature $[8,11,12]$. Ethics committee approval was not necessary.

\section{Surgical technique}

\section{TO approach}

The TOET was performed under general anesthesia and in the supine position, with neck extension. A diluted epinephrine solution (1:200,000) was injected into the oral vestibule area. A $2.5-\mathrm{cm}$ oral mucosal incision was then made in the gum on the lip side of the incisor teeth.

The mucoperiosteum was cut and separated along the labial surface of the bone to the inferior border of the mandible. Blunt dissection using a curved tunneler was performed up to the medial border of both sternocleidomastoid muscles laterally and the suprasternal notch inferiorly. Subsequently, another two incisions were made near the turning point of the mucosa of the vestibular sulcus, and bilateral instrumental tunnels were formed using a $0.5-\mathrm{cm}$ trocar and intersected at the hyoid plane. A $1.0-\mathrm{cm}$ trocar with an observation lens was then inserted. Subsequently, carbon dioxide $\left(\mathrm{CO}_{2}\right)$ with a pressure of $6 \mathrm{~mm} \mathrm{Hg}$ and flow rate of 12 l/min was inflated. Flap dissection was performed using a harmonic scalpel. The flap was freed at the deep surface of the platysma, allowing the bilateral sides to reach the medial edge of the sternocleidomastoid muscle from the hyoid to the suprasternal plane. After establishing the working space (Photo $1 \mathrm{~A}$ ), the ceiling flap was suspended at the planes of the cricoid cartilage and hyoid bone using two No. 2-0 sutures. The $\mathrm{CO}_{2}$ pressure was then decreased to $4 \mathrm{~mm} \mathrm{Hg}$ to reduce the risk of gas embolism, and dissection was made at the strap muscles using a harmonic scalpel. The strap muscles were then pulled laterally using a retractor to expose the thyroid gland (Photo $1 \mathrm{~B}$ ), and the pyramidal lobe was filed to transect the isthmus. Part of the sternothyroid muscle was transected to expose the upper pole of the thyroid. The thyroid gland, including the nodules, was then removed. The RLN and parathyroid gland were identified, and a tunnel was opened on the nerve surface (Photo $1 \mathrm{C}$ ). The medial lymph nodes and adipose tissue of the RLN were then freed at the medial side (Photo $1 \mathrm{D}$ ), and the operating cavity was rinsed with sterile water. Finally, the surgical incision was closed, and a slender drainage tube was placed in one of the instrumental tunnels in the bed of the thyroid gland $[11,12]$.

\section{BA approach}

Under general anesthesia, a 1-cm vertical incision was made next to the medial border of the right are- 

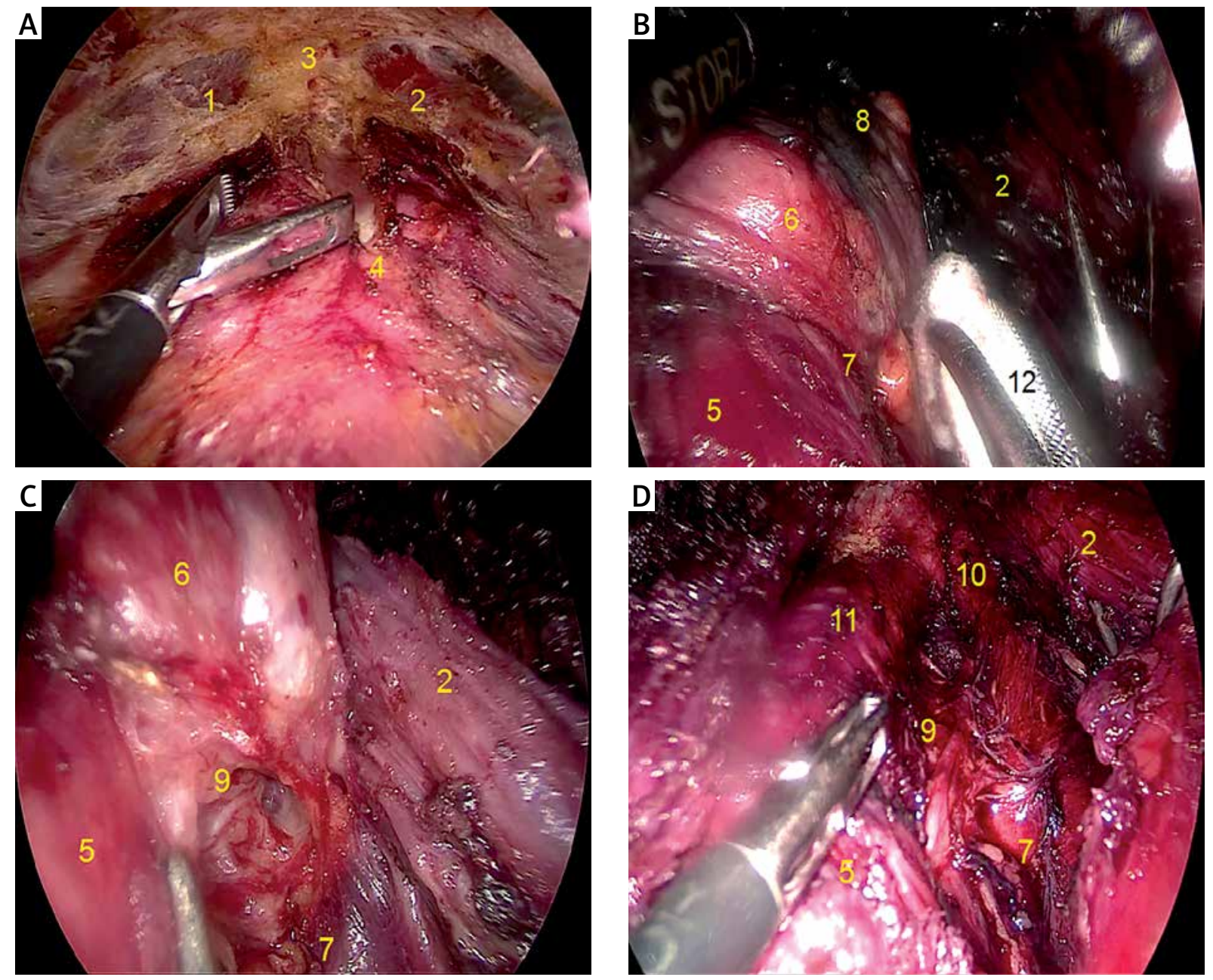

Photo 1. Surgical procedure in TO approach. 1 - Left sternocleidomastoid, 2 - right sternocleidomastoid, 3 - suprasternal fossa, 4 - linea alba cervicalis, 5 - cricothyroid muscle, 6 - thyroid gland, 7 - superior parathyroid, 8 - inferior parathyroid, 9 - recurrent laryngeal nerve (RLN), 10 - common carotid artery, 11 - trachea, 12 - an absorbable gelatin sponge used to separate and stop bleeding

ola, and a diluted epinephrine solution $(1: 200,000)$ was injected into the observation tunnel and manubrium. A surgical space was made using a stripping rod prod to both sternoclavicular joints. Subsequently, a 5-mm incision was made at the left and right areolar borders to produce instrumental tunnels. The inflation of $\mathrm{CO}_{2}$ was the same as that used in the TO approach. In the BA approach, the bilateral sides of the flap reached the middle part of the sternocleidomastoid muscle up to the hyoid plane (Photo 2 A). The isthmus was then transected (Photo 2 B). The method of removing the thyroid lobe was the same as that used in the TA group. The anterior tracheal lymph node was then lifted to the outside, and a careful search was conducted for the RLN in the lymphoid adipose tissue. The RLN was then pushed to the tracheal side (Photo $2 \mathrm{C}$ ). Subsequently, the lymph nodes were pulled to the outside and removed (Photo 2 D). Finally, the operating cavity was flushed, and a drainage tube was placed in one of the instrumental tunnels.

\section{Comparison of the TA and BA approaches}

A single surgeon performed all the TO and BA surgical procedures. The following parameters were compared in the two groups: the total operative time; central neck dissection time; intraoperative blood loss; superior parathyroid preservation in situ; inferior parathyroid preservation in situ; operative bleeding; postoperative drainage; cosmetic effects; 

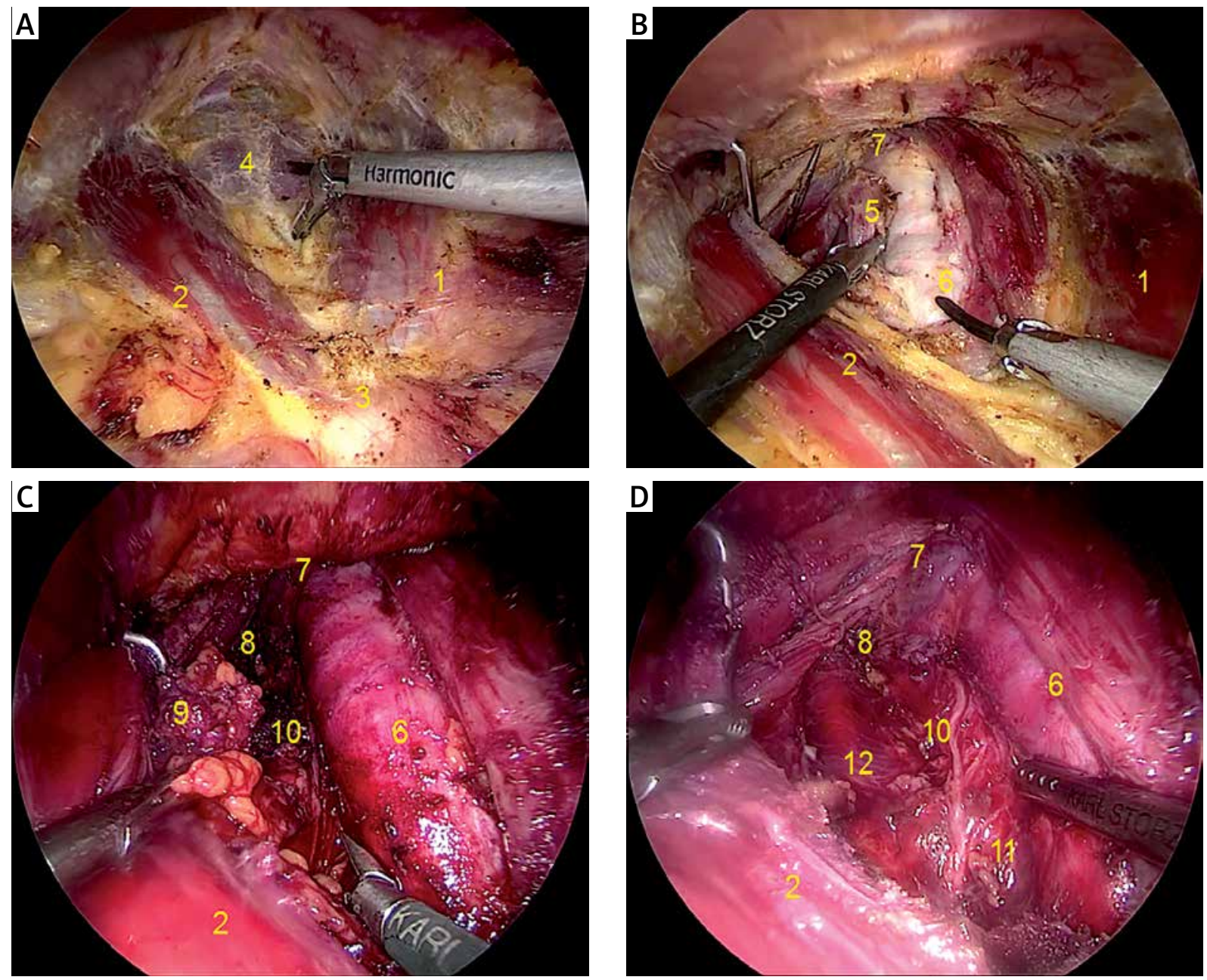

Photo 2. Surgical procedure in the BA approach. 1 - Left sternocleidomastoid, 2 - right sternocleidomastoid, 3 - suprasternal fossa, 4 - linea alba cervicalis, 5 - isthmus of thyroid gland, 6 - trachea, 7 - cricothyroid muscle, 8 - superior parathyroid, 9 - central lymphoid adipose tissue, 10 - recurrent laryngeal nerve $(\mathrm{RLN}), 11$ - inferior parathyroid, 12 - common carotid artery

operative complications, including swallowing discomfort; transient RLN palsy; transient hypoparathyroidism; permanent RLN palsy; and duration and costs of hospitalization.

The total operative time was determined from the time of the skin incision to closure, and central neck dissection time was determined as the time from the start of lymph node dissection until lymph node removal $[13,14]$. The cosmetic effect was assessed using a subjective satisfaction scale questionnaire, with the satisfaction score classified as minimum 0 (very dissatisfied) to maximum 10 (very satisfied). Ultrasound and an electronic laryngoscope were used to evaluate the activity of the vocal cords once the patient appeared hoarse, and vocal cord activity was reassessed using an electronic laryngoscope 3 and 6 months after surgery. The hospitalization duration was defined as the time from the day of surgery to discharge. Drainage was used in all the operations. The drain was removed when the drain volume was less than $20 \mathrm{ml}$ per day, and the patient was discharged 1 day after the drainage tube was removed. The Kruskal-Wallis test and $\chi^{2}$ test were used for measurement and categorical data, respectively.

\section{Statistical analysis}

For all statistical analyses, a value of $p<0.05$ was accepted as the standard for statistical significance. All data were processed using SPSS 19.0 software. 


\section{Results}

The clinical characteristics and details of the surgical parameters in both the TO and BA groups are summarized in Table I. The ages of the patients (male: $n=9$; female: $n=83$ ) ranged from 19 to 49 years, with a median age of 32 years. There were no significant between-group differences in the age and sex of the patients or the side, body mass index, total operative time, operative bleeding, and superior parathyroid preservation in situ (all $p>0.05$ ). The mean central neck dissection time in the TO group was lower than that in the BA group $(31.4 \pm 4.0 \mathrm{~min}$ vs. $39.5 \pm 4.6 \mathrm{~min}, p<0.01)$. The number of central lymph nodes resected in the TO group was higher than that in the BA group ( $8.6 \pm 3.7$ vs. $5.4 \pm 2.3, p=$ $0.017)$. The amount of postoperative drainage in the TO group was lower than that in the BA group (47.2 $\pm 17.1 \mathrm{ml}$ vs. $63.4 \pm 19.3 \mathrm{ml}, p<0.01$ ). The extubation time was significantly shorter in the TO group than that in the BA group $(2.7 \pm 0.6$ days vs. $3.0 \pm 0.4$ days, $p=0.043)$. Inferior parathyroid preservation in situ in the TO group was lower than that in the BA group (20 vs. 30, $p=0.013$ ). However, there was no statistically significant between-group difference in superior parathyroid preservation in situ (38 vs. 29, $p=0.168)$.

The postoperative characteristics and hospitalization duration and costs in both groups are summarized in Table II. There were no significant differences in operative complications, including swallowing discomfort, RLN palsy, and hypoparathyroidism (all $p>0.05$ ). The cosmetic effect in the TO group was better than that in the BA group $(9.2 \pm 0.7$ vs. $7.7 \pm 1.3, p<0.01)$. The hospitalization costs in the TO group were higher than those in the BA group $(3,912.4 \pm 134.8$ U.S. dollars vs. 3,851.1 \pm 123.8 U.S. dollars, $p=0.026)$, but there was no statistically significant difference in the hospital duration $(3.7 \pm 0.5$ days vs. $3.9 \pm 0.4$ days, $p=0.097$ ).

\section{Discussion}

A TOET can be performed via a vestibular approach or via the floor of the mouth. Due to the low risk of complications and ease of implementation, the vestibular approach has developed rapidly in recent years $[15,16]$.

In this study, there was no statistically significant difference in the ages of the patients in the two groups. In the present study, most of the unmarried female patients preferred the TOET approach, which has a better cosmetic effect $(p<0.01)$, over the BA approach. The TOET approach also does not damage the galactophore.

In the present study, although there were no significant between-group differences in the total operative time $(p=0.065)$, the central neck dissection time in the TO group was lower than that in the BA group. Based on our experience, it is easier to estab-

Table I. Clinical characteristics and details of surgical parameters of the patients

\begin{tabular}{|lccc|}
\hline Characteristics & TO group $(n=48)$ & BA group $(n=44)$ & $P$-value \\
\hline Age [years] & $30.46 \pm 6.93$ & $33.3 \pm 6.94$ & 0.055 \\
\hline Gender (male/female) & $4 / 44$ & $5 / 39$ & 0.732 \\
\hline Side (left/right) & $23 / 25$ & $20 / 24$ & 0.837 \\
\hline BMI [kg/m²] & $22.5 \pm 3.2$ & $22.3 \pm 3.2$ & 0.737 \\
\hline Total operative time [min] & $107.2 \pm 11.8$ & $102.6 \pm 11.8$ & 0.065 \\
\hline Central neck dissection time [min] & $31.4 \pm 4.0$ & $39.5 \pm 4.6$ & $<0.01$ \\
\hline Operative hemorrhage $[\mathrm{ml}]$ & $21.5 \pm 5.8$ & $22.4 \pm 6.1$ & 0.501 \\
\hline Postoperative drainage $[\mathrm{ml}]$ & $47.2 \pm 17.1$ & $63.4 \pm 19.3$ & $<0.01$ \\
\hline Extubation time [day] & $2.7 \pm 0.6$ & $3.0 \pm 0.4$ & 0.043 \\
\hline Number of central lymph nodes resected & $8.6 \pm 3.7$ & $5.4 \pm 2.3$ & 0.017 \\
\hline Superior parathyroid preserved in situ be discovered & 38 & 29 & 0.168 \\
\hline Inferior parathyroid preserved in situ be discovered & 20 & 30 & 0.013 \\
\hline
\end{tabular}


Table II. Postoperative characteristics and length and costs of hospitalization

\begin{tabular}{|c|c|c|c|}
\hline Characteristics & TO group $(n=48)$ & BA group $(n=44)$ & $P$-value \\
\hline Cosmetic effect & $9.2 \pm 0.7$ & $7.7 \pm 1.3$ & $<0.01$ \\
\hline Swallowing discomfort & 3 & 2 & 1 \\
\hline Transient recurrent laryngeal nerve palsy & 1 & 3 & 0.346 \\
\hline Permanent recurrent laryngeal nerve palsy & 0 & 0 & \\
\hline Temporary hypocalcemia & 5 & 4 & 1 \\
\hline Permanent hypocalcemia & 0 & 0 & \\
\hline Postoperative bleeding & 1 & 0 & 1 \\
\hline Postoperative infection & 1 & 1 & 1 \\
\hline Postoperative day of discharge [day] & $3.7 \pm 0.5$ & $3.9 \pm 0.4$ & 0.097 \\
\hline Costs of hospitalization [dollars] & $3912.4 \pm 134.8$ & $3851.1 \pm 123.8$ & 0.026 \\
\hline
\end{tabular}

lish a working space and expose the thyroid gland, as well as separate the linea alba and isthmus, using the BA approach. Conversely, full exposure of the RLN is easier and faster using the TO approach, thereby making en bloc removal of the central lymph node much easier. Thus, the time consumption was shorter than in the BA group $(p<0.01)$. Furthermore, the risk of traction injury to the RLN was minimized in the TO group. In the TO group, the RLN was first found near Berry's ligament. Berry's ligament was then freed, the RLN was dissected downward, and the thyroid was lifted to the upward side. In the BA group, the central lymph nodes were pulled laterally from the trachea, thereby increasing the risk of traction injury to the RLN. Simultaneously, separating the nerves in lymphoid adipose tissue increased the risk of thermal damage. Although there was no between-group difference in the risk of transient RLN palsy ( $p=0.346)$, further prospective long-term studies should be performed.

When performing central lymph node dissection, the innominate artery was clearly visible in the TO group (Photo $1 \mathrm{D}$ ), allowing lymph nodes between the trachea and thymus to be easily removed. Using the TO procedure, even upper mediastinal tumors and lymph nodes can be removed. In contrast, when performing an ET via the BA approach, the central lymph nodes cannot be completely dissected due to blockage of the sternum (Photo 2 D) $[17,18]$. In the present study, the number of central lymph nodes resected in the TO group was higher than that in the BA group $(p=0.017)$.
The location of the upper parathyroid gland is relatively constant. In the TO group, the superior parathyroid gland was revealed by cutting off the upper thyroid lobe and lifting the upper pole. Conversely, the inferior parathyroid was often obscured by the thyroid lobe in the TO group (Photo $1 \mathrm{~B}$ ), and preservation of the inferior parathyroid in situ was easier in the BA group than in the TO group ( $p=0.013)$, although there was no between-group difference in superior parathyroid preservation in situ $(p=0.168)$. We believe that the TOET approach has more advantages in protection of the superior parathyroid glands, which is more important in a thyroidectomy. Further clinical research needs to be undertaken to shed light on this issue. For all the above reasons, some surgeons advocate en bloc resection of the thyroid and central lymph nodes in a TOET. Then look for the parathyroid gland in the specimen and use the injection method to inject the chopped parathyroid gland into the sternocleidomastoid muscle.

The dissection area of the flap in the TO group was smaller than that in the BA group, resulting in less postoperative drainage in the TO group as compared with that in the BA group $(p<0.01)$. The extubation time was also shorter in the TO group $(p=0.043)$.

In the TO approach, the incision is made in the mouth, which increases the risk of infection. Thus, an oral preparation, including a chlorhexidine and metronidazole compound, should be administered 3 days prior to the surgery, and antibiotics should be used to prevent infection during the perioperative 
period. In the present study, there was no statistically significant between-group difference in postoperative infection $(p=1)$. However, due to the additional use of antibiotics in the TO group, the costs of hospitalization were higher in this group as compared with those in the BA group $(p=0.026)$.

One of the major concerns associated with a TOET is mental nerve palsy [19]. In the current study, transient numbness of the lower lip occurred in 22 of the 48 patients in the TO group, with 16 cases occurring in the first 30 cases. However, all the patients recovered in 1-3 months. To reduce the swing and traction of the mental nerve when using a trocar, we use a vascular clamp attached to the periosteum to enlarge bilateral instrumental tunnels. This significantly reduces the incidence of transient numbness of the lower lip.

The present study included a few male patients. As the thyroid cartilage of male patients is bulkier than that of female patients, it is especially important for surgeons to use both hands alternately when performing a TOET. As the anterior chest skin of male patients is too tight to lift the surgical instruments, the surgical incision should be made in the third intercostal space.

The TOET approach has some disadvantages: It is difficult to operate in the high superior pole, and the working space is too small to handle a large goiter, where the mass exceeds $5 \mathrm{~cm}$. Furthermore, using this approach, it is difficult to remove specimens through a $2.5-\mathrm{cm}$ oral mucosal incision. Thus, the incision needs to be extended along the two sides of the vestibular sulcus. In addition, a modified radical neck dissection cannot be performed using the TO approach [19, 20].

The present study has some limitations. This was a comparative study of TO and BA ET approaches performed by a single surgeon. However, in terms of which procedure was chosen, some selection bias may exist, as this was a prospective study, not a blind randomized study. In addition, in our center, the BA approach to an ET has been implemented for 4 years, whereas the TOET approach has been implemented for a shorter period (i.e., nearly 2 years). Thus, our center has more experience in ET via the BA approach than the TO approach.

\section{Conclusions}

Both TO and BA approaches are safe and effective techniques for performing an ET. We recommend the former when the patient requires central neck dissection. The BA approach may be a better choice when the PTC mass is located in the high superior pole or the mass is a large benign tumor.

\section{Conflict of interest}

The authors declare no conflict of interest.

\section{References}

1. Siegel RL, Miller KD, Jemal A. Cancer statistics, 2019. CA Cancer J Clin 2019; 69: 7-34.

2. Davies L, Welch HG. Current thyroid cancer trends in the United States. JAMA Otolaryngol Head Neck Surg 2014; 140: 317-22.

3. La Vecchia C, Malvezzi M, Bosetti C, et al. Thyroid cancer mortality and incidence: a global overview. Int J Cancer 2015; 136 : 2187-95.

4. Davies L, Welch HG. Current thyroid cancer trends in the United States. JAMA Otolaryngol Head Neck Surg 2014; 140: 317-22.

5. Macedo FI, Mittal VK. Total thyroidectomy versus lobectomy as initial operation for small unilateral papillary thyroid carcinoma: a meta-analysis. Surg Oncol 2015; 24: 117-22.

6. Donatini G, Castagnet M, Desurmont T, et al. Partial thyroidectomy for papillary thyroid microcarcinoma: is completion total thyroidectomy indicated. World J Surg 2016; 40: 510-5.

7. Chen W, Zheng R, Baade PD, et al. Cancer statistics in China, 2015. CA Cancer J Clin 2016; 66: 115-32.

8. Bian C, Liu H, Yao XY, et al. Complete endoscopic radical resection of thyroid cancer via an oral vestibule approach. Oncol Lett 2018; 16: 5599-606.

9. Kang SW, Lee SC, Lee SH, et al. Robotic thyroid surgery using a gasless, transaxillary approach and the da Vinci S system: the operative outcomes of 338 consecutive patients. Surgery 2009; 146: 1048-55.

10. Tan Z, Gu J, Han Q, et al. Comparison of conventional open thyroidectomy and endoscopic thyroidectomy via breast approach for papillary thyroid carcinoma. Int J Endocrinol 2015; 2015: 239610.

11. Kim WW, Lee J, Jung JH, et al. A comparison study of the transoral and bilateral axillo-breast approaches in robotic thyroidectomy. J Surg Oncol 2018; 118: 381-7.

12. Anuwong A. Transoral endoscopic thyroidectomy vestibular approach: a series of the first 60 human cases. World J Surg 2016; 40: 491-7.

13. Liu N, Chen B, Li L, et al. Subplatysmal or subfascial approach in totally endoscopic thyroidectomy has better postoperative efficacy for voice, sensory, swallowing symptoms and cosmetic result. Cohort study. Int J Surg 2018; 60: 22-7.

14. Kim SJ, Lee KE, Myong JP, et al. Recovery of sensation in the anterior chest area after bilateral axillo-breast approach endoscopic/robotic thyroidectomy. Surg Laparosc Endosc Percutan Tech 2011; 21: 366-71.

15. Zhao L, Li JY, Wang CC. The research progress of endoscopic thyroidectomy through the mouth. Chin J Surg 2014; 52: 541-3. 
16. Russell JO, Clark J, Noureldine SI, et al. Transoral thyroidectomy and parathyroidectomy - a North American series of robotic and endoscopic transoral approaches to the central neck. Oral Oncol 2017; 71: 75-80.

17. Yang J, Wang C, Li J, et al. Complete endoscopic thyroidectomy via oral vestibular approach versus areola approach for treatment of thyroid diseases. J Laparoendosc Adv Surg Tech A 2015; 25: 470-6.

18. Witzel K, Hellinger A, Kaminski C, et al. Endoscopic thyroidectomy: the transoral approach. Gland Surg 2016; 5: 336-41.

19. Dionigi G, Tufano RP, Russell J, et al. Transoral thyroidectomy: advantages and limitations. J Endocrinol Invest 2017; 40: 1259-63.

20. Anuwong A, Ketwong K, Jitpratoom P, et al. Safety and out comes of the transoral endoscopic thyroidectomy vestibular approach. JAMA Surg 2018; 153: 21-7.

Received: 12.02.2019, accepted: 17.04.2019. 